\title{
Optical Characteristics of Polystyrene Based Solid Polymer Composites: Effect of Metallic Copper Powder
}

\author{
Shujahadeen B. Aziz, Sarkawt Hussein, Ahang M. Hussein, and Salah R. Saeed \\ Department of Physics, Faculty of Science, University of Sulaimani, Kurdistan Regional Government, Sulaimani City, Iraq \\ Correspondence should be addressed to Shujahadeen B. Aziz; shujaadeen78@yahoo.com
}

Received 3 September 2013; Revised 28 October 2013; Accepted 28 October 2013

Academic Editor: Mohamed Bououdina

Copyright (C) 2013 Shujahadeen B. Aziz et al. This is an open access article distributed under the Creative Commons Attribution License, which permits unrestricted use, distribution, and reproduction in any medium, provided the original work is properly cited.

\begin{abstract}
Solid polymer composites (SPCs) were prepared by solution cast technique. The optical properties of polystyrene doped with copper powder were performed by means of UV-Vis technique. The optical constants were calculated by using UV-Vis spectroscopy. The dispersion regions were observed in both absorption and refractive index spectra at lower wavelength. However, a plateau can be observed at high wavelengths. The small extinction coefficient compared to the refractive index reveals the transparency of the composite samples. The refractive index and optical band gap were determined from the reflectance and optical absorption coefficient data, respectively. The nature of electronic transition from valence band to conduction band was determined and the energy band gaps of the solid composite samples were estimated. It was observed that, upon the addition of $\mathrm{Cu}$ concentration, the refractive index increased while the energy gaps are decreased. The calculated refractive indexes (low index of refraction) of the samples reveal their availability in waveguide technology.
\end{abstract}

\section{Introduction}

Recent years have witnessed constant search for high permittivity materials that have wide range of technologically important applications such as microelectronic, embedded passive, and electrostrictive devices. The majority of the electronic components in microelectronic circuits are passive and occupy more than $80 \%$ of the printed wired surface area [1]. The dispersion of an electrically conductive phase within an insulating polymer matrix affects the overall performance of the heterogeneous system. It was reported that if the dispersed metallic particle is in sufficient quantity, a conductive or semiconductive composite is formed. The interesting properties of such systems make them technologically important and competitive to other alternative materials due to their cost-effectiveness [2]. Conductive polymer composites are essential for applications referring to electromagnetic interference (EMI) shielding, radio frequency interference (RFI) shielding, and electrostatic dissipation of charges (ESD). Polymer composites are used as electrical conductive adhesives and circuit elements in microelectronics and have been reported to possess anticorrosive behavior as metal components coatings [3]. Many types of polymer composites have been studied in the pursuit to develop a system with high conductivity. These include epoxy resin matrix with iron particles [2], high-density polyethylene (HDPE) with multiwalled carbon nanotubes (MWNTs) [4], poly(pphenylene vinylene) (PPV)- $\mathrm{TiO}_{2}$ [5], and polyvinylidene fluoride (PVDF) with multiwalled carbon nanotube (MWCNT) [6]. Current studies reveal that the measurement and understanding of the optical properties of materials are important. The occurrence of electronic transitions in the structure of materials is directly related to the photon energy. The nature of the highest occupied molecular orbital (HOMO) and the lowest unoccupied molecular orbital (LUMO) of the linear and cyclic alkanes was understood through the study of the absorbance edge and index of refraction [7]. Nonpolar polymers have the lowest dielectric constant of any known solid polymer, and this makes them attractive for demanding electronics applications. According to Yang at al., polymers that have the lowest index of refraction are very suitable as low-index claddings for waveguide applications [8]. Optical characterization of thin films gives information about some important physical properties, such as band gap energy 
and band structure and role of defects, and therefore may be of permanent interest for several different applications. The widely used envelop method has been developed for transmittance measurements to evaluate the refractive index, extinction coefficient, and absorption coefficient [9]. The extensive and intensive survey of the literature reveals that there is a very little work that has been done on the optical analysis of polymers containing metallic particles. Hence, the goal of this work is to study the optical parameters of polymer composites based on polystyrene-copper system.

\section{Experimental Detail}

2.1. Sample Preparation. Polystyrene (procured from Sigma) and copper powders from sigma (with sizes of microns) have been used as the raw materials in this work to prepare the solid polymer composites (SPCs) using the solution cast technique. For this purpose $1 \mathrm{gm}$ of polystyrene was dissolved in $25 \mathrm{~mL}$ of toluene solution. The mixture was stirred continuously with a magnetic stirrer for several hours at room temperature until the polystyrene has completely dissolved. While the above systems were still in the liquid state, various amounts of copper powder were added for the production of the solid composite samples. The copper powder content in the prepared samples was varying from $0 \mathrm{wt} . \%$ to $6 \mathrm{wt} .0 \%$ in volume fraction and the mixtures were stirred continuously until homogeneous solutions were obtained. The solutions were then cast into different clean and dry glass Petri dish and allowed to evaporate at room temperature until solventfree films were obtained. The films were kept in desiccators with silica gel desiccant for further drying. Table 1 shows the concentration of the prepared samples.

2.2. UV-Vis Measurement. The UV-Vis spectra of the solid polymer composites based on polystyrene have been recorded using UV-Vis spectra (model: Lambda 25) in the absorbance mode. To calculate the transmittance $(T)$, absorption coefficient $(\alpha)$, extinction coefficient $(k)$, and refractive index $(n)$, the optical study was performed for polystyrene- $x \mathrm{Cu}(0.01 \leq x \leq 0.06)$ composites by analyzing the absorption spectra as follows [7, 9-11]:

$$
\begin{gathered}
T_{\text {sample }}=10^{-A}, \\
\alpha=\frac{-1}{t} \ln (T), \\
k=\frac{\alpha \times \lambda}{4 \pi t}, \\
n=\frac{(1+\sqrt{R})}{(1-\sqrt{R})},
\end{gathered}
$$

where $T_{\text {sample }}$ is the amount of light transmitted through the sample, $A$ is the optical absorbance (base 10) of the sample, $\alpha$ is absorption coefficient, $t$ is the sample thickness, $\lambda$ is the wavelength, and $k$ is the extinction coefficient. $R$ is the reflectance $R=(1-(A+T))$ of the sample which is used to estimate the refractive index. Equation (2) is valid for low loss
TABLE 1: Composition of PS : $x \mathrm{Cu}(0.01 \leq x \leq 0.06)$ solid polymer composites (SPCs).

\begin{tabular}{lccc}
\hline Designation polystyrene $(\mathrm{g})$ & Cu powder (wt. \%) & Cu powder $(\mathrm{g})$ \\
\hline $\mathrm{SPC}_{1}$ & 1.0 & 0.0 & 0.0000 \\
$\mathrm{SPC}_{2}$ & 1.0 & 1.0 & 0.0101 \\
$\mathrm{SPC}_{3}$ & 1.0 & 2.0 & 0.0204 \\
$\mathrm{SPC}_{4}$ & 1.0 & 3.0 & 0.0309 \\
$\mathrm{SPC}_{5}$ & 1.0 & 5.06 & 0.0626 \\
\hline
\end{tabular}

materials, that is, small extinction coefficient $(k)$. The optical absorption coefficient was used to determine the energy band gap of the solid polymer composites, which is the most direct and simplest method, using the following relationship [12]:

$$
\alpha h v=B\left(h v-E_{g}\right)^{m},
$$

where $B$ is an energy-independent constant, $E_{g}$ is the optical energy band gap, and $m$ is a constant which determines the nature of the optical transition from the valence band to the conduction band (fundamental absorption). The nature of the transition can be determined by determining the value of $m$ which is, respectively, $1 / 2$ and 2 for allowed direct and indirect transitions.

\section{Results and Discussion}

The optical absorption and transmission spectra may provide some insight into the optical behavior of the samples. Figures 1 and 2 show the absorbance and transmittance spectra for all the samples, respectively. It can be noticed that upon the addition of copper powders the absorption increases very rapidly at $6 \mathrm{wt} . \%$, while the transmittance decreased. It is obvious that the absorption and transmission spectra of the samples (Figures 1 and 2) with wavelength are in sharp manner, that is, nonexponential. The sharp behavior of absorbance and transmittance with wavelength for all the samples indicates the crystalline nature of the samples [13]. According to these results the behavior of absorption coefficient $(h \alpha \nu)$ versus photon energy $(\mathrm{eV})$ should be nonexponential.

Figures 3 and 4 show the refractive index and extinction coefficient as a function of wavelengths. It can be seen that the refractive indexes of the samples are larger than their extinction coefficients. The small extinction coefficient $\left(\approx 10^{-5}\right)$ indicates that the composite samples still are very transparent [14]. Study of refractive index and the extinction coefficient for optical systems performance are important. The index of refraction is crucial for matching or optimizing the numerical aperture (NA) of the reduction optical system at all points in the optical path. The extinction coefficient is critical to determine optical losses in the system [15]. The extinction coefficient is the fraction of electromagnetic energy lost due to scattering and absorption per unit thickness in a particular medium. The variation of $n$ and $k$ values with wavelength reveals that some interaction takes place between photons and electrons. The increase of extinction coefficient at high wavelengths (Figure 4) is related to the higher concentration of copper powders (from 2 to $6 \mathrm{wt} \% \%$ of $\mathrm{Cu}$ powder) and 


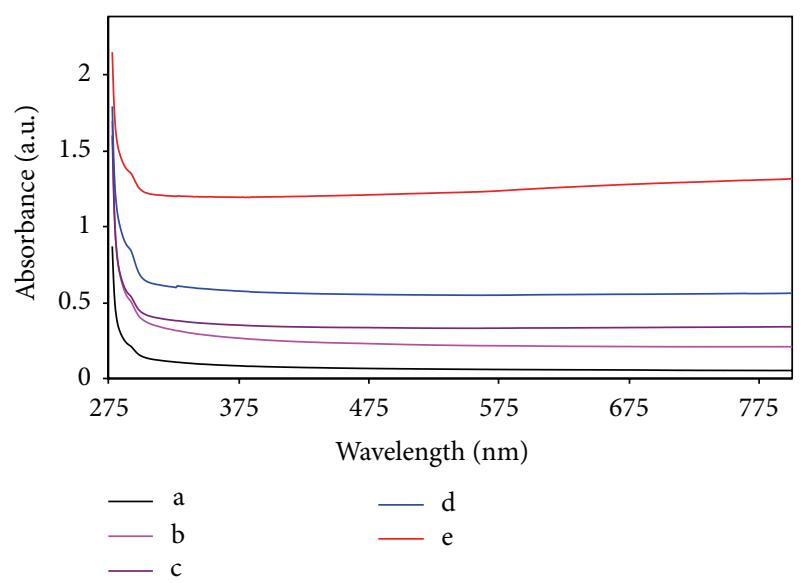

FIgURE 1: Absorption spectra as a function of wavelength for (a) $\mathrm{SPC}_{1}$, (b) $\mathrm{SPC}_{2}$, (c) $\mathrm{SPC}_{3}$, (d) $\mathrm{SPC}_{4}$, and (e) $\mathrm{SPC}_{5}$.

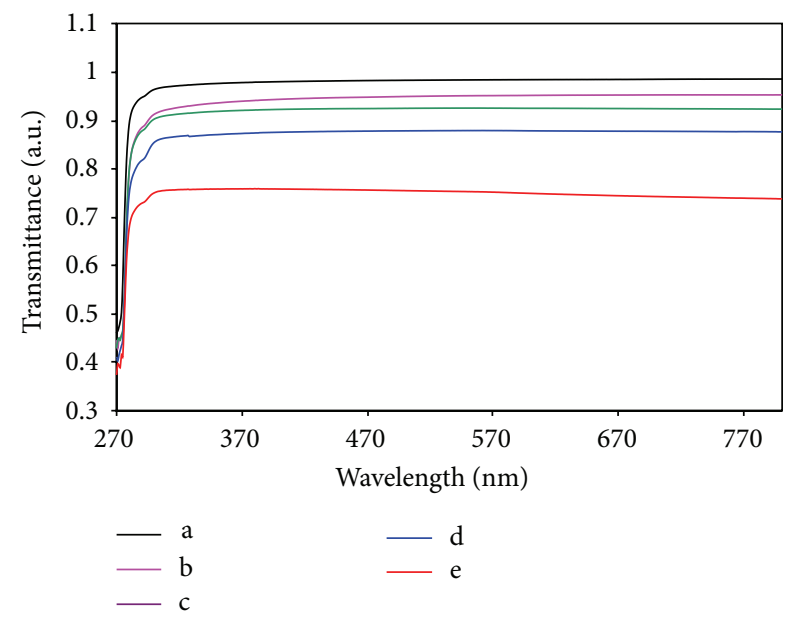

FIGURE 2: Transmittance spectra as a function of wavelength for (a) $\mathrm{SPC}_{1}$, (b) $\mathrm{SPC}_{2}$, (c) $\mathrm{SPC}_{3}$, (d) $\mathrm{SPC}_{4}$, and (e) $\mathrm{SPC}_{5}$.

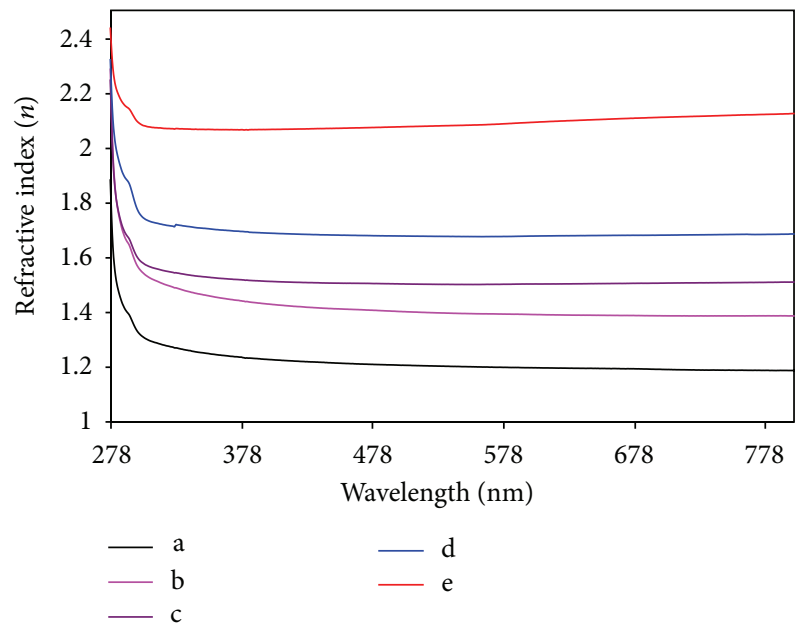

FIGURE 3: Refractive index spectra as a function of wavelength for (a) $\mathrm{SPC}_{1}$, (b) $\mathrm{SPC}_{2}$, (c) $\mathrm{SPC}_{3}$, (d) $\mathrm{SPC}_{4}$, and (e) $\mathrm{SPC}_{5}$.

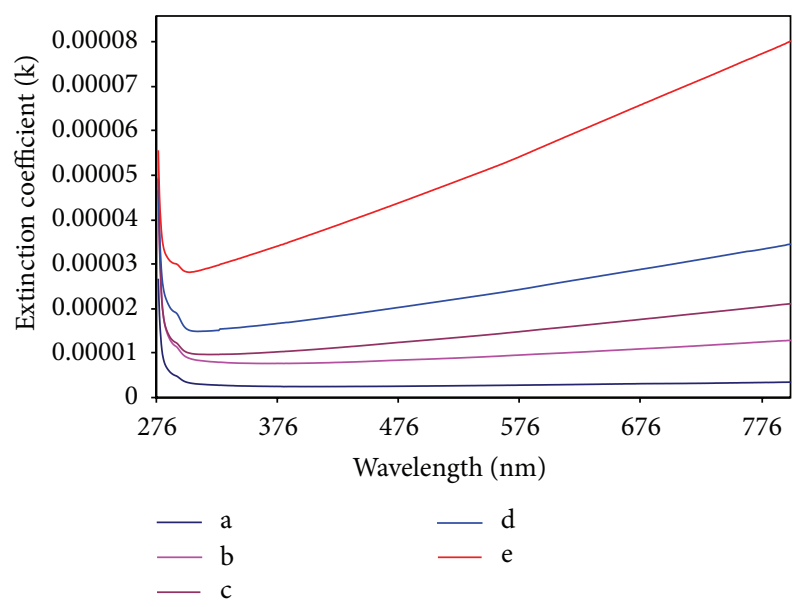

FIGURE 4: Extinction coefficient spectra as a function of wavelength for (a) $\mathrm{SPC}_{1}$, (b) $\mathrm{SPC}_{2}$, (c) $\mathrm{SPC}_{3}$, (d) $\mathrm{SPC}_{4}$, and (e) $\mathrm{SPC}_{5}$.

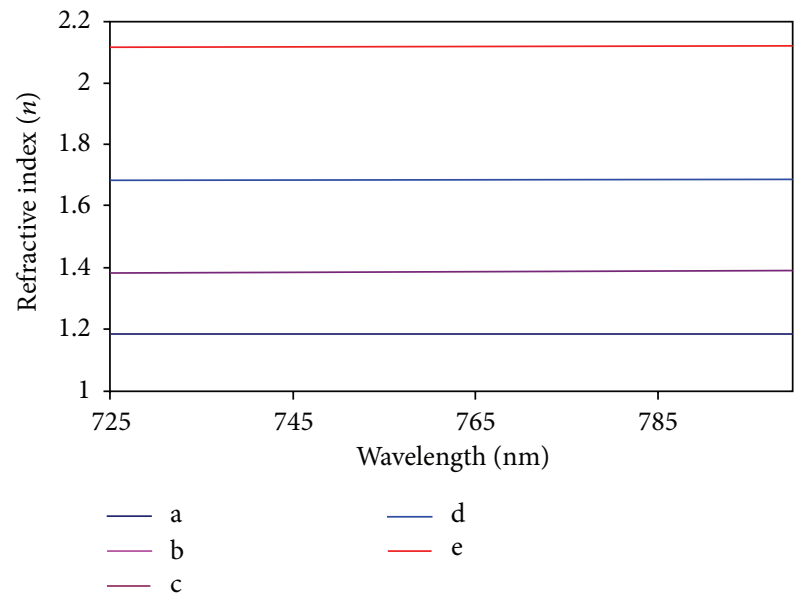

FIGURE 5: High wave length region of refractive index spectra as a function of wavelength for (a) $\mathrm{SPC}_{1}$, (b) $\mathrm{SPC}_{2}$, (c) $\mathrm{SPC}_{3}$, (d) $\mathrm{SPC}_{4}$, and (e) $\mathrm{SPC}_{5}$.

thus more scattering of photons are occurred with the added $\mathrm{Cu}$ powder. The change in refractive index and extinction coefficient with wavelength of the incident light beam is due to the above mentioned interactions [16].

It can be seen that the refractive index (Figure 3 ) increases with increasing copper powder, that is, the refractive index of the composite samples is tunable upon the addition of copper powder concentration. The high wavelength region of refractive index represents the material (bulk) property and is almost independent on the wavelength as depicted in Figure 5. The sudden increase of refractive index at $6 \mathrm{wt} . \%$ may be attributable to percolation threshold phenomena. It is well known that when the content of conductive particle reaches to a critical value, that is, a percolation threshold, a continuous network can be formed by these conductive particles and thus increasing the crystallinity [17]. The sharply behavior of $\left((h \alpha v)^{\wedge 2}\right)$ versus photon energy $(h v)$ at higher $\mathrm{Cu}$ powder concentration can completely confirm the dominant 


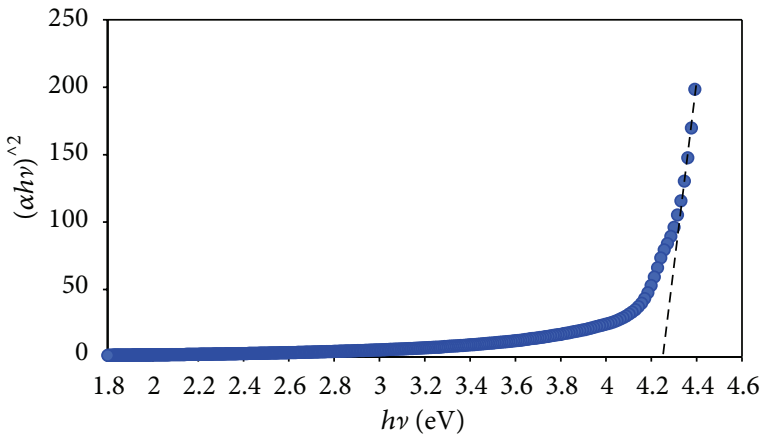

(a)

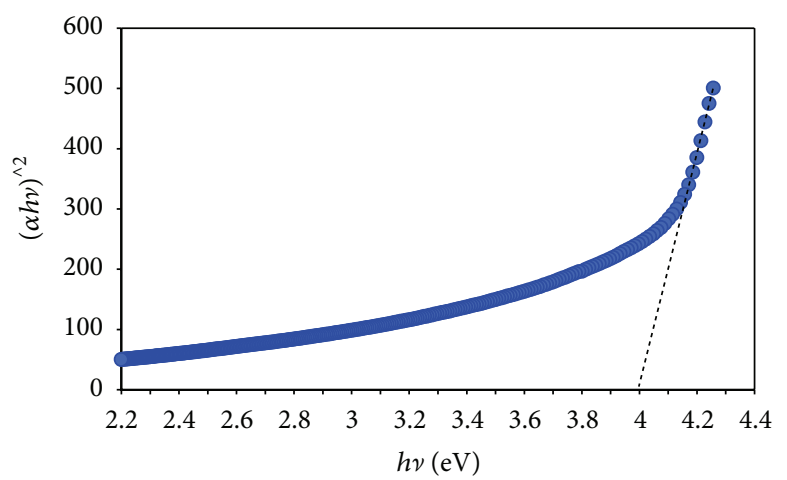

(c)

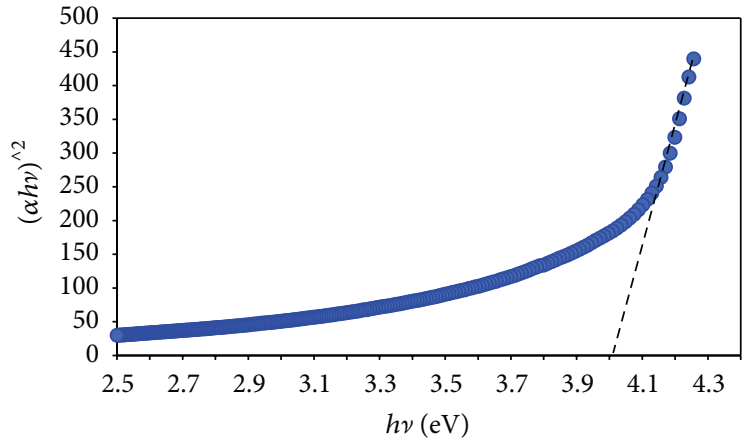

(b)

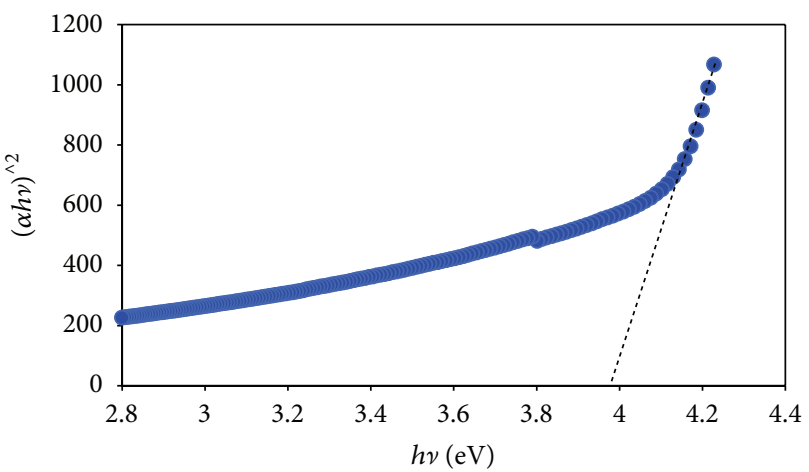

(d)

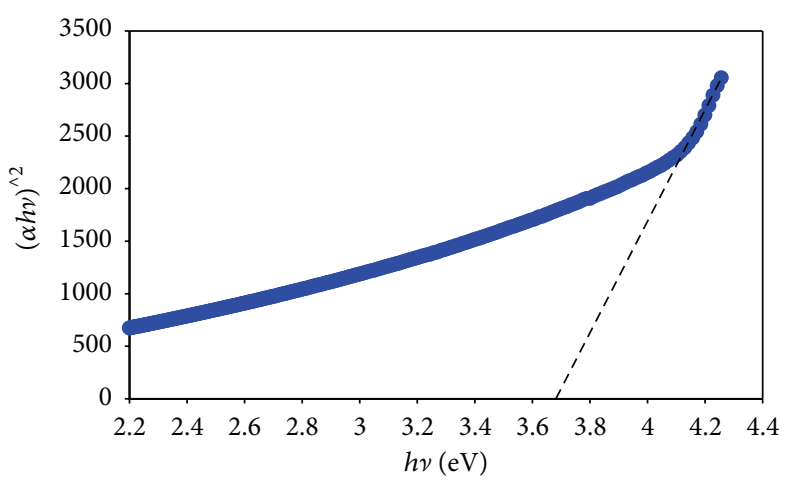

(e)

FIGURE 6: The plot of $(h \alpha v)^{\wedge 2}$ versus $h v$ for (a) $\mathrm{SPC}_{1}$, (b) $\mathrm{SPC}_{2}$, (c) $\mathrm{SPC}_{3}$, (d) $\mathrm{SPC}_{4}$, and (e) $\mathrm{SPC}_{5}$.

of crystalline portion in the samples as can be seen in later sections.

From the refractive index (Figure 5) study in this work we conclude that the energy gap of the samples may be altered upon the addition of copper powder. This can be more understood through the study of energy band gap and refractive index as a function of copper concentration. For this purpose the plot of absorption coefficient $\left((h \alpha \nu)^{\wedge 2}\right)$ as a function of photon energy $(h \nu)$ enable us to calculate the energy band gaps. In order to show the influence of copper powder on the optical band gap of the solid polymer composite films, the optical absorption edge was investigated for all the samples. Figures $6(\mathrm{a})-6(\mathrm{e})$ show the dependence of $(h \alpha \nu)^{\wedge 2}$ versus $h v$ for all the samples. The comprehensible absorption edge shown by all the samples establish the dominant of crystalline nature of the samples [18]. It is evident that the absorption edge shifted towards the lower energy side upon the addition $\mathrm{Cu}$ powder especially at $6 \mathrm{wt} . \%$ of $\mathrm{Cu}$. These results confirm the fact that the energy band gap and the refractive index are strongly correlated.

The direct energy band gaps for all samples were determined from the dotted lines intersection on the photon energy axis of Figures 6(a)-6(e) and plotted as a function of copper concentration as depicted in Figure 7. It is obvious that the energy band gap is steeply decreased at $6 \mathrm{wt} . \%$ of $\mathrm{Cu}$ powder. One possible interpretation of this experimental observation is that $\mathrm{Cu}$ powder introduces multiple valence states in the polystyrene structure and thus decreasing the band gap energy between the valence and conductions bands. 


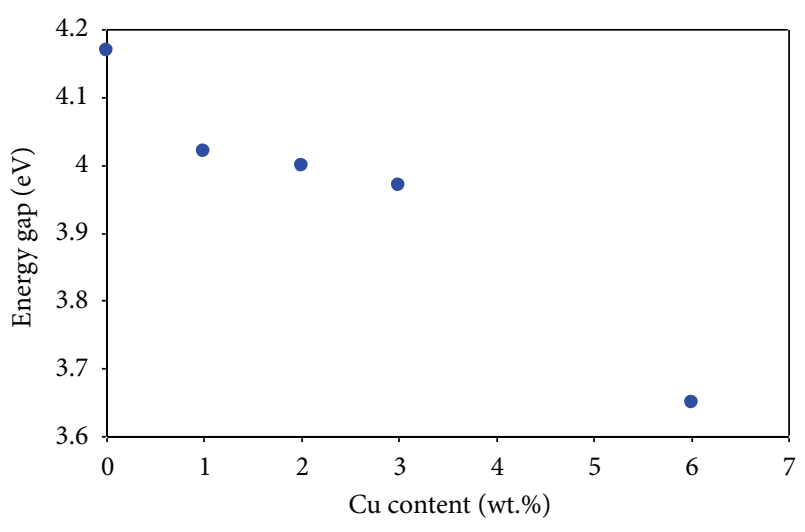

FIGURE 7: Energy gap as a function of copper concentration (wt.\%).

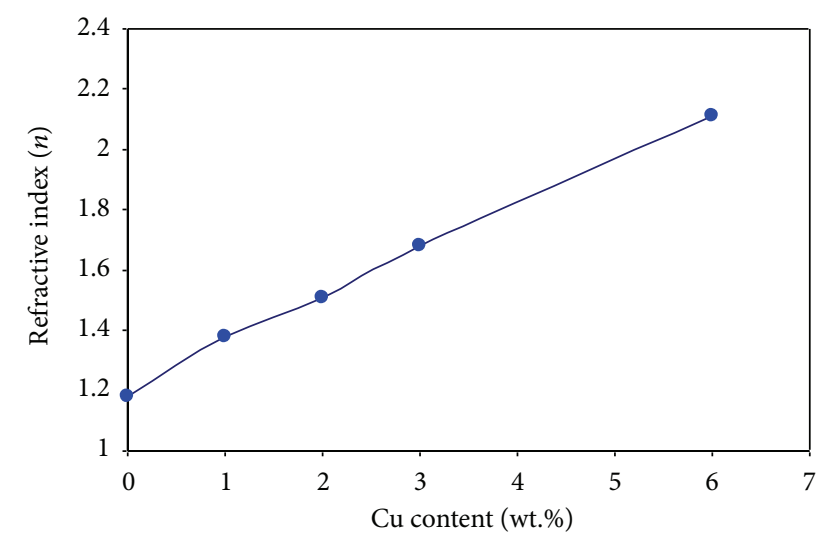

FigURE 8: Refractive index as a function of copper concentration (wt.\%).

Variation of refractive index as a function of copper concentration permits us to understand the behavior of energy gap variation with copper concentration. Figure 8 shows the copper powder dependence of refractive index which calculated from the intersection of the plateau of Figure 5 on the $Y$-axis. It is clear that the energy band gap (Figure 7) and the refractive index (Figure 8) follows the same trend but in a different manner. It was reported that the optical properties (index of refraction and energy gap) of a material are related to the variation in material composition and atomic arrangement [19]. The small value of the refractive indexes (1.2 to 2.18) obtained in this work reveals that PS based polymer composites are very suitable as low-index claddings for waveguide applications [8].

\section{Conclusion}

The dispersion region in both absorption and refractive indexes at lower wavelength can be ascribed to the sufficient time for polarization with the electric field component of the electromagnetic light. A plateau of absorption and refractive indexes at high wavelengths may be attributable to the inertia of the side groups of polystyrene which is difficult when following the electric field alternation. The calculated energy band gaps of the solid composite samples show that the nature of electronic transition from valence band to conduction band was a direct transition. Upon the addition of $\mathrm{Cu}$ concentration, the refractive index increased from 1.2 up to 2.1 and the energy gap decreased from 4.05 to about $3.65 \mathrm{eV}$ as a result of the introduction of more multiple states. The small refractive indexes of PS based composites indicate their importance in waveguide application. This work confirms that the refractive index and energy gap are strongly correlated.

\section{Acknowledgment}

The authors gratefully acknowledge the Ministry of Higher Education and Scientific Research, Kurdistan Regional Government, University of Sulaimani, for the financial support.

\section{References}

[1] S. George and M. T. Sebastian, "Three-phase polymer-ceramicmetal composite for embedded capacitor applications," Composites Science and Technology, vol. 69, no. 7-8, pp. 1298-1302, 2009.

[2] G. C. Psarras, E. Manolakaki, and G. M. Tsangaris, "Dielectric dispersion and ac conductivity in-iron particles loaded: polymer composites," Composites A, vol. 34, no. 12, pp. 1187-1198, 2003.

[3] G. C. Psarras, "Hopping conductivity in polymer matrix-metal particles composites," Composites A, vol. 37, no. 10, pp. 15451553, 2006.

[4] F. Liu, X. Zhang, W. Li et al., "Investigation of the electrical conductivity of HDPE composites filled with bundle-like MWNTs," Composites A, vol. 40, no. 11, pp. 1717-1721, 2009.

[5] S. H. Yang, T. P. Nguyen, P. le Rendu, and C. S. Hsu, "Optical and electrical properties of $\mathrm{PPV} / \mathrm{SiO}_{2}$ and $\mathrm{PPV} / \mathrm{TiO}_{2}$ composite materials," Composites A, vol. 36, no. 4, pp. 509-513, 2005.

[6] Q. Li, Q. Xue, L. Hao, X. Gao, and Q. Zheng, "Large dielectric constant of the chemically functionalized carbon nanotube/polymer composites," Composites Science and Technology, vol. 68, no. 10-11, pp. 2290-2296, 2008.

[7] E. A. Costner, B. K. Long, C. Navar et al., "Fundamental optical properties of linear and cyclic alkanes: VUV absorbance and index of refraction," Journal of Physical Chemistry A, vol. 113, no. 33, pp. 9337-9347, 2009.

[8] M. K. Yang, R. H. French, and E. W. Tokarsky, "Optical properties of Teflon AF amorphous fluoropolymers," Journal of Micro/ Nanolithography, MEMS, and MOEMS, vol. 7, no. 3, Article ID 033010, pp. 1-9, 2008.

[9] M. Caglar, Y. Caglar, and S. Ilican, "The determination of the thickness and optical constants of the $\mathrm{ZnO}$ crystalline thin film by using envelope method," Journal of Optoelectronics and Advanced Materials, vol. 8, no. 4, pp. 1410-1413, 2006.

[10] A. Tadjarodi, M. Imani, and H. Kerdari, "Application of a facil solid state process to synthesize the $\mathrm{CdO}$ spherical nanoparticles," International Nano Letters, vol. 3, article 43, 2013.

[11] N. A. Hamizi and M. R. Johan, "Optical properties of CdSe quantum dots via non-TOP based route," International Journal of Electrochemical Science, vol. 7, pp. 8458-8467, 2012.

[12] F. Yakuphanoglu, M. Sekerci, and A. Balaban, "The effect of film thickness on the optical absorption edge and optical constants 
of the Cr(III) organic thin films," Optical Materials, vol. 27, no. 8, pp. 1369-1372, 2005.

[13] N. Ahlawat, S. Sanghi, A. Agarwal, and S. Rani, "Effect of $\mathrm{Li}_{2} \mathrm{O}$ on structure and optical properties of lithium bismosilicate glasses," Journal of Alloys and Compounds, vol. 480, no. 2, pp. 516-520, 2009.

[14] L. Bi, A. R. Taussig, H.-S. Kim et al., "Structural, magnetic, and optical properties of $\mathrm{BiFeO}_{3}$ and $\mathrm{Bi}_{2} \mathrm{FeMnO}_{6}$ epitaxial thin films: an experimental and first-principles study," Physical Review B, vol. 78, no. 10, Article ID 104106, 2008.

[15] R. H. French, J. M. Rodríguez-Parada, M. K. Yang, R. A. Derryberry, and N. T. Pfeiffenberger, "Optical properties of polymeric materials for concentrator photovoltaic systems," Solar Energy Materials \& Solar Cells, vol. 95, no. 8, pp. 20772086, 2011.

[16] T. Arumanayagam and P. Murugakoothan, "Studies on optical and mechanical properties of new organic NLO crystal: guanidinium 4-aminobenzoate (GuAB)," Materials Letters, vol. 65, no. 17-18, pp. 2748-2750, 2011.

[17] T. N. Zhou, X. D. Qi, and Q. Fu, "The preparation of the poly(vinyl alcohol)/grapheme nanocomposites with low percolation threshold and high electrical conductivity by using the large-area reduced grapheme oxide sheets," Express Polymer Letters, vol. 7, no. 9, pp. 747-755, 2013.

[18] K. C. Preetha, K. V. Murali, A. J. Ragina, K. Deepa, and T. L. Remadevi, "Effect of cationic precursor $\mathrm{pH}$ on optical and transport properties of SILAR deposited nano crystalline $\mathrm{PbS}$ thin films," Current Applied Physics, vol. 12, no. 1, pp. 53-59, 2012.

[19] X. Rocquefelte, S. Jobic, and M.-H. Whangbo, "Concept of optical channel as a guide for tuning the optical properties of insulating materials," Solid State Sciences, vol. 9, no. 7, pp. 600$603,2007$. 

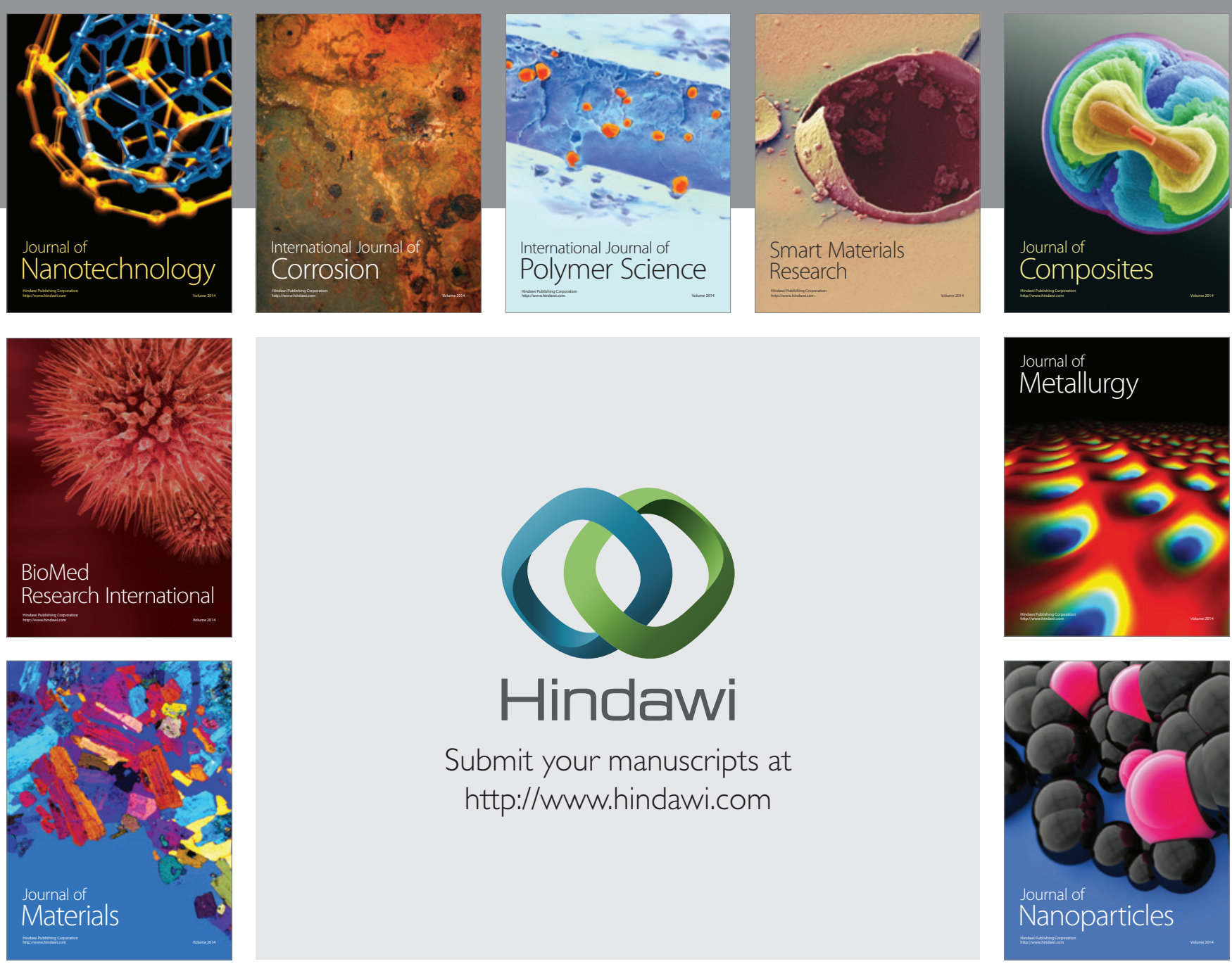

Submit your manuscripts at http://www.hindawi.com
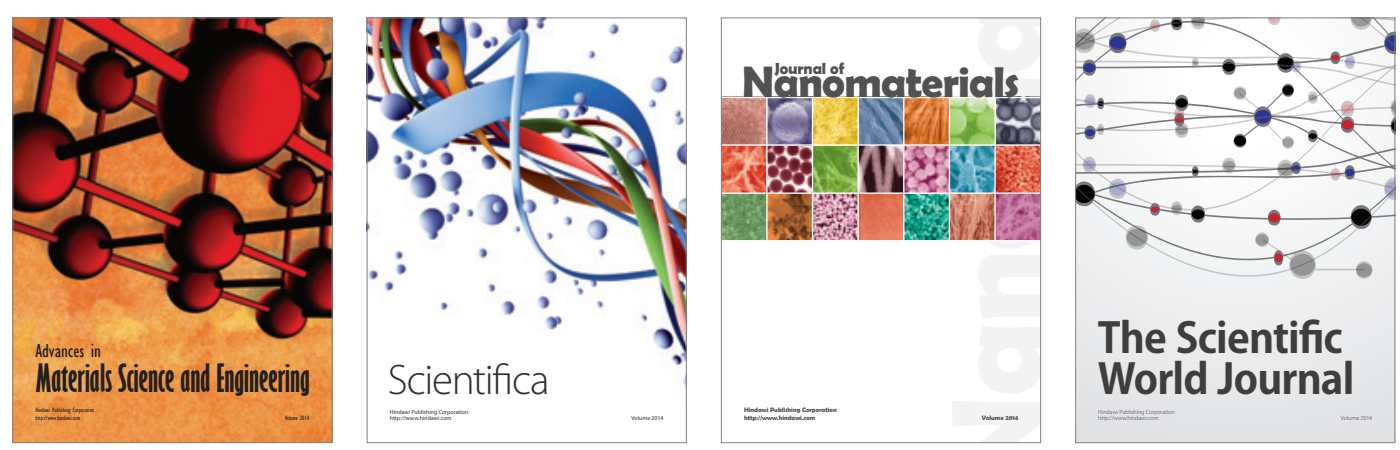

\section{The Scientific World Journal}
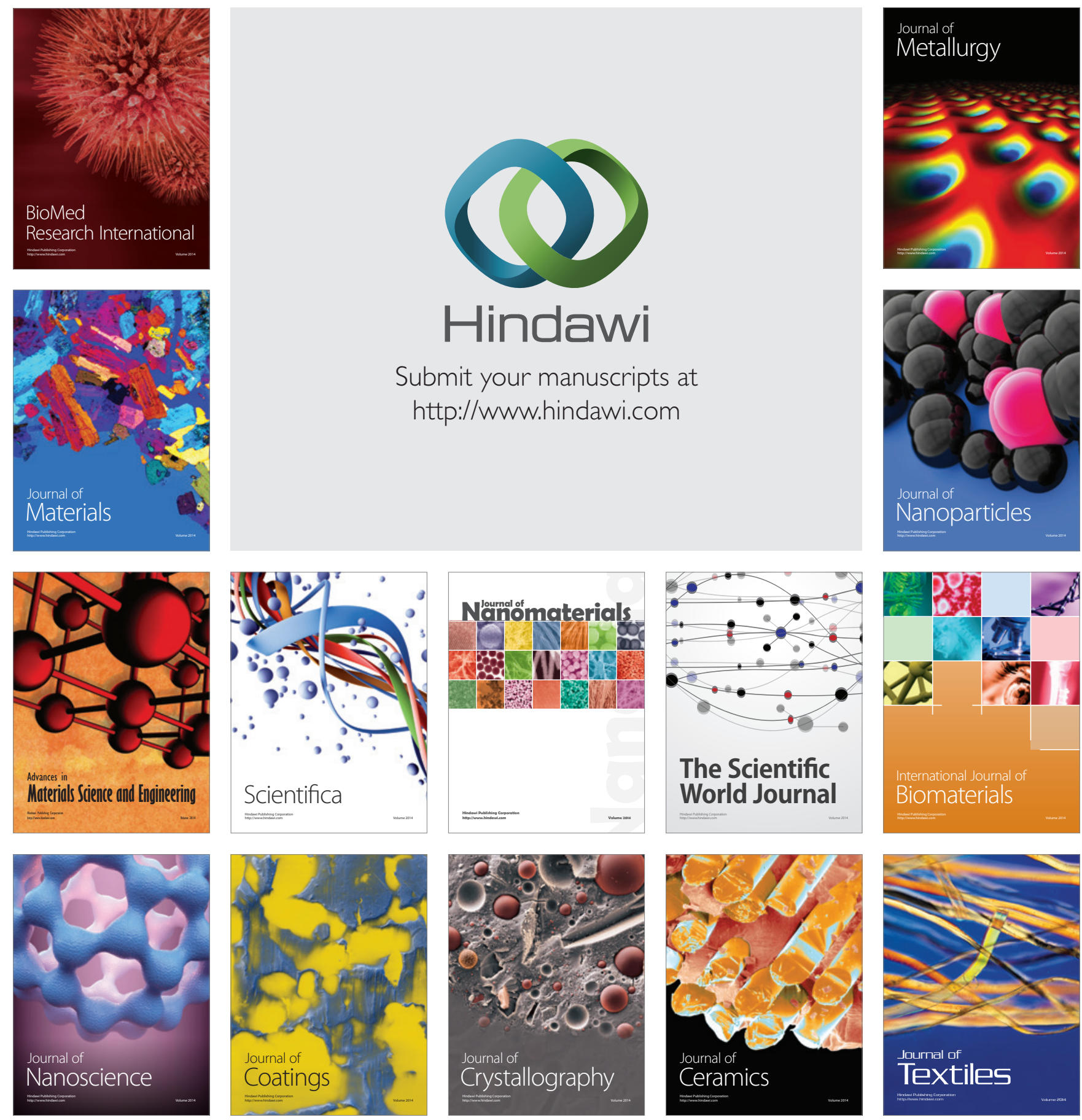